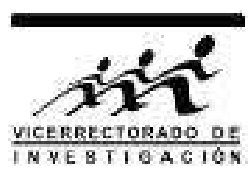

\title{
Red de grafeno artificial en sistemas de electrones 2D de GaAs/AIGaAs
}

\author{
D. I. Arrieta, J. M. Marchena, R. A. Montalvo, J. W. Flores y P. H. Rivera* \\ Facultad de Ciencias Físicas, Universidad Nacional Mayor de San Marcos, Lima, Perú
}

Recibido 30 mayo 2013 - Aceptado 15 setiembre 2013

El grafeno, desde el año 2004, ha mostrado unas excepcionales propiedades mecánicas, térmicas y electrónicas en sus diferentes configuraciones como monocapas, bicapas, puntos cuánticos, nanoribbons y superredes. Las potenciales aplicaciones van desde moduladores ópticos, transistores, detectores de gas, dispositivos electrocrómicos, electrodos, disipadores térmicos hasta circuitos integrados.

Existe un inconveniente en cuanto a la manipulación átomo por átomo para obtener las propiedades específicas de cada función en cada dispositivo. Entre las que se puede contar el desorden en la estructura del grafeno y las impurezas que se intercalan en la estructura hexagonal y las rugosidades de los substratos que permite unas ondulaciones en la estructura del grafeno. Sin embargo, no permiten modificar la formación del cono entre la banda de valencia y la banda de conducción. El punto de Dirac prevalece.

$\mathrm{Se}$ ha sugerido otras opciones para inducir un gap en los puntos de simetría $\mathrm{K}$ y $\mathrm{K}^{\prime}$ donde se encuentran los puntos de Dirac en la que convergen los dos conos de los portadores de carga, electrones y huecos.

Una de ellas es la de construir una red artificial hexagonal sobre un gas bidimensional de electrones que se encuentra en la interface de los materiales semiconductores de GaAs/AIGaAs.

Por tanto, el objetivo del presente trabajo es verificar si la red artificial hexagonal emula las propiedades electrónicas del grafeno, es decir, verificar la existencia de los puntos de Dirac en una red hexagonal artificial. Para ello hemos realizado un estudio sistemático de la estructura electrónica mediante la aproximación tight-binding para simular la estructura de la red hospedera que nos proporciona el mínimo de la banda de conducción del GaAs y sobre ella simular una red hexagonal de antipuntos cuánticos que nos permite visualizar en detalle la evolución de la estructura electrónica de los mismos respecto a un campo magnético aplicado perpendicularmente al plano de los sitios de la red hospedera.

Palabras claves: Estructura electrónica del grafeno, magnetoresistencia, transporte electrónico en el grafeno. PACS: 73.22.Pr, 73.43.-f, 72.80.Vp.

\section{Artifical graphene lattice in 2D electron systems in GaAs/AIGaAs}

The graphene, since 2004, had show some exceptional mechanical, thermal and electronic properties in several configurations such as optical modulators, transistors, gas detectors, electrocromic devices, electrodes, thermal dissipaters and integrated circuits.

There is an inconvenient in the atom by atom manipulation for obtain the specific properties for each function in each proposed device. We account between them, the disorder in the graphene structure and the intercalation of impurities in the hexagonal structure and the imperfections of the substrate that incorporate ripples in the graphene structure. Nevertheless, these do not modify the cones of the valence and conduction bands. The Dirac point remains.

Other options has been suggested to induce a gap between the $\mathrm{K}$ and $\mathrm{K}^{\prime}$ symmetry points where lay the Dirac points in which converge the two charge carrier cones, electrons and holes.

One of them is to build an artificial hexagonal lattice over a two dimensional electron gas which found near to the interface of the two semiconductor material with different gaps, GaAs/AIGaAs.

Therefore, the objective of the present work is to verify if the artificial hexagonal lattice simulate the electronic properties of graphene, i.e., to verify the existence of Dirac points in an artifical hexagonal lattice.

Keywords: Graphene electronic structure, magnetoresistence, electronic transport on graphene.

*priverar@unmsm.edu.pe 
La estructura electrónica del grafeno en los puntos de simetría $\mathrm{K}$ y $\mathrm{K}^{\prime}$ presenta una intersección puntual entre las bandas de valencia y conducción que se denominan los puntos de Dirac, en la que degeneran los huecos y electrones. Los dos conos formados corresponden a la banda de valencia y de conducción, ambos presentan una dependencia lineal respecto a la magnitud del vector de onda y a una velocidad de Fermi que medido experimentalmente es del orden de $10^{6} \mathrm{~m} / \mathrm{s}$ [1 2]. Lo que porporciona una mobilidad de $10,000 \mathrm{~cm}^{2} / \mathrm{V}$-s [3] a temperatura ambiente y $45,000 \mathrm{~cm}^{2} / \mathrm{V}$-s [2] a $1.7 \mathrm{~K}$.

Para el diseño de dispositivos optoelectrónicos es necesario inducir un gap en los puntos $\mathrm{K}$ y $\mathrm{K}^{\prime}$, para ello se ha sugerido el confinamiento espacial en una dimensión, dirección zigzag, el cual se consigue a través de los nanoribbons de grafeno que se orientan en la dirección armchair. Conforme se reduce el tamaño a lo largo de la dirección zigzag se induce un gap en dichos puntos, que se denomina una transición semimetal-semiconductor [4] 5].

Cuando se produce un confinamiento en las dos direcciones -un punto cuántico de grafeno-, no se consigue una transición semimetal-semiconductor, pero existe un acoplamiento muy intenso entre los estados de borde con los estados de bulk que nos permite identificar, claramente, con la aplicación simultánea de un campo magnético y un campo AC, la evolución de una estructura electrónica semejante a la estructura electrónica de un anillo cuántico cuando la dirección del campo AC es paralelo a la dirección armchair [6] y la dirección del campo magnético perpendicular al plano del grafeno.

Otra forma de inducir gaps en los puntos $\mathrm{K}_{\text {y }} \mathrm{K}^{\prime}$ es construir redes de antipuntos cuánticos en el grafeno [7], esto se obtiene quitando átomos de carbono con determinada geometría y con determinado parámetro de red, mediante litografía.

Una nueva alternativa es la creación de una red artificial de grafeno sobre materiales semiconductores que posean un gap directo en el punto $\Gamma[8-10]$, esto es, verificar que la estructura hexagonal construída litográficamente sobre el GaAs/AIGaAs, como una red de antipuntos cuánticos, imita las extraordinarias propiedades electrónicas que posee el grafeno. Esta alternativa es el objetivo del presente estudio que ha continuación detallamos.

\section{El modelo}

Cuando se realiza el crecimiento epitaxial del GaAs y AlGaAs sobre un substrato de GaAs, la ionización de los electrones en el Al que se encuentran en la capa de AlGaAs permite el surgimiento de un gas bidimensional de electrones en el GaAs, muy próximo a la interface de ambos materiales; este gas bidimensional de electrones posee unas propiedades de transporte bastante extraordinarias que han sido objeto de un estudio intensivo durante los últimos treinta años.

Se han realizado mediante litografía diferentes diseños de redes de antipuntos para explorar las propiedades electrónicas del gas bidimensional con potenciales artificiales creados mediante la litografía [11]12].

Desde el punto de vista de la física computacional, se debe emular el fondo de la banda de conducción del GaAs. Existe técnicas bastante conocidas y precisas para calcular la estructura electrónica del GaAs/AIGaAs como el método de los pseudopotenciales. Diseñar en esas técnicas el efecto de los potenciales de los antipuntos incluyendo el efecto de campo magnético y campo AC, es una proeza computacionalmente muy pesada.

Por ello, recurrimos a técnicas más simples para emular el fondo de la banda de conducción del GaAs, usando las propiedades conocidas y calculadas del GaAs, como es el método tight-binding. Usamos esta técnica en el esquema de la aproximación de la masa efectiva que nos permite calcular el acoplamiento entre los sitios heurísticos que emulan la banda de conducción del GaAs. Consideramos que el fondo de la banda de conducción, puede ser simulado por una red rectangular de sitios heurísticos que poseen un parámetro de red heurístico de aproximadamente $20 \AA$, con orbitales tipo $s$ que se acoplan mediante un parámetro de hopping dado por

$$
V_{x, y}=-\frac{\hbar^{2}}{2 m^{*} a_{x, y}^{2}}
$$

donde la masa efectiva de los electrones en el GaAs es $m^{*}=0.067 m_{e}$ siendo $m_{e}$ la masa del electrón, $a_{x}=$ $a_{y}=a=20 \AA$, el parámetro de red heurístico y $\hbar$ es la constante de Planck dividido por $2 \pi$.

El hamiltoniano que emula la banda de conducción del GaAs como una red rectangular con sitios heurísticos a primeros vecinos con orbitales tipo $s$ está dado por

$$
\begin{array}{r}
H=\sum_{i j} E_{i j}^{(0)}+\sum_{i j} V_{x}[|i, j\rangle\langle i+1, j|+| i+1, j\rangle\langle i, j|]+ \\
V_{y}[|i, j\rangle\langle i, j+1|+| i, j+1\rangle\langle i, j|]
\end{array}
$$

donde $E_{i j}^{(0)}$ son las energías de los orbitales tipo $s$ de los sitios heurístico indexados por los pares coordenados $(i, j)$; $V_{x}$ e $V_{y}$ son los parámetros de hopping a lo largo de la dirección $x$ e $y$, advirtiendo que $i$ y $j$ indican la discretización en las direcciones $x$ e $y$, respectivamente.

En la figura 1 se muestra un esquema de la porción de la red heurística emulada. 


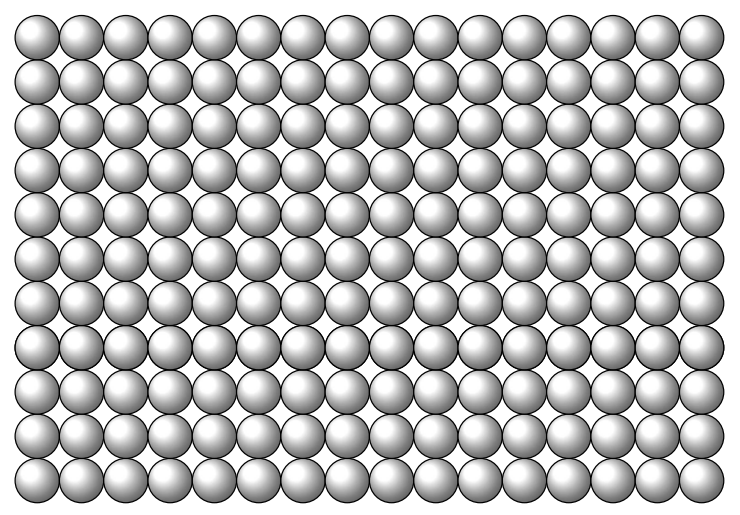

Figura 1: Red rectangular de sitios heurísticos que emulan el fondo de la banda de conducción del GaAs.

Para simular una red artificial de grafeno usando el fondo de una red rectangular que emula la banda de conducción del GaAs, debemos generar la red hexagonal en la red rectangular. Para esto hemos creado un algoritmo que nos permite obtener aproximadamente una red hexagonal. Este diseño de la red hexagonal lo hacemos via la generación de los antipuntos, variando la energía del orbital tipo $s$ de algunas regiones del fondo de la red rectangular (puntos azules) tal como se muestra en la figura 2.

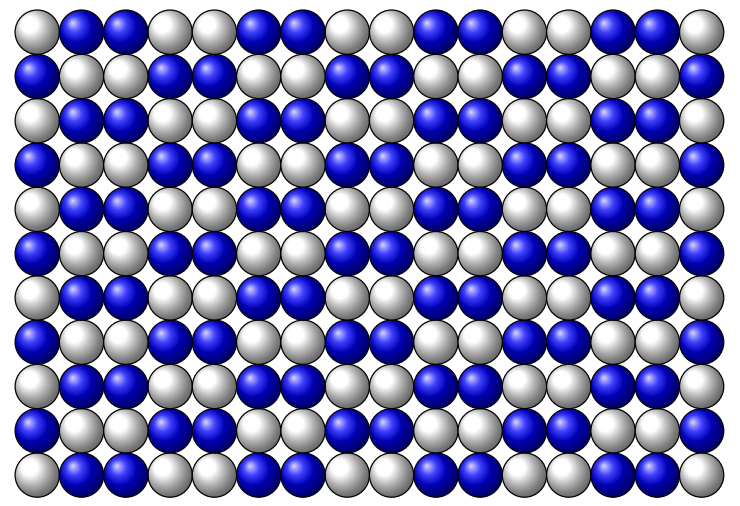

Figura 2: Red hexagonal de antipuntos (azules) simulado en una red rectangular (blancos).

Se observa que la dirección armchair de la red hexagonal coincide con el eje $x$, mientras que, la dirección zigzag coincide con la dirección $y$ de la red rectangular.

Para analizar la estructura electrónica aplicamos un campo magnético perpendicular al plano de la red heurística de modo que el efecto del campo se incorpora en el hamiltoniano, Ec.(2), a través de la aproximación de Peierls y usando el gauge de Landau para el potencial vectorial magnético, el efecto del campo magnético se incorpora multiplicando la fase magnética al término $V_{y}$ de modo que resulta $V_{y} e^{i 2 \pi p / q}$, donde $p$ y $q$ son números racionales que describe la razón entre el flujo magnético por celda unitaria de la red heurística $\Phi=B A$ y el quan- tum de flujo magnético para un electrón $\Phi_{e}=h / e$, donde $A=a_{x} a_{y}$ es el área de la celda unitaria de la red hospedera, $B$ el campo magnético, $h$ la constante de Planck y $e$ la carga del electrón.

Por otro lado, la distribución de la corriente de probabilidad es una herramienta que nos permite determinar el flujo de corriente de probabilidad en el dispositivo. Para nuestro análisis, consideramos que el efecto Zeeman del campo magnético sobre los espínes electrónicos son muy pequeños y no contribuyen significativamente a la determinación del flujo de las corrientes de probabilidades. Por tanto, la corriente de probabilidad de un sistema en la presencia de un potencial vectorial magnético está dado por

$$
\boldsymbol{J}=\frac{\hbar}{m^{*}}\left(\operatorname{Im}\left[\psi^{*} \nabla \psi\right]-\frac{q}{\hbar} \boldsymbol{A}|\psi|^{2}\right)
$$

Para un sistema cristalino bidimensional, como el nuestro, usamos el gauge de Landau para el potencial vectorial magnético $\boldsymbol{A}=(0, x B, 0)$, luego las componentes de la corriente de probabilidad están dados por

$$
\begin{aligned}
& J_{x}=\frac{\hbar}{m^{*}}\left(\operatorname{Im}\left[\psi^{*} \frac{\partial \psi}{\partial x}\right]\right) \\
& J_{y}=\frac{\hbar}{m^{*}}\left(\operatorname{Im}\left[\psi^{*} \frac{\partial \psi}{\partial y}\right]-\frac{q}{\hbar} x B|\psi|^{2}\right),
\end{aligned}
$$

considerando que la autofunción de onda $\psi(\boldsymbol{r})=\psi(x, y)$.

Para los electrones, la parte dependiente del potencial vectorial se expresa como

$$
\begin{aligned}
-\frac{q}{\hbar} x B|\psi|^{2}=-2 \pi \frac{-e}{h} x B|\psi|^{2} & = \\
2 \pi \frac{x B}{h / e}|\psi|^{2} & =2 \pi \frac{\imath a_{x} B}{\Phi_{e}}|\psi|^{2}
\end{aligned}
$$

donde $\imath$ es el índice de los sitios en la dirección $x, \Phi_{e}$ es la unidad de flujo magnético cuantizado para un electrón. Al expresar la dimensión $x=\imath a_{x}$ estamos discretizando la longitud a lo largo de la dirección $x$. Las derivadas espaciales también son discretizadas usando las diferencias finitas, para ello consideramos también, $y=\jmath a_{y}$. Luego

$$
\partial x=a_{x} \partial \imath \quad \text { y } \quad \partial y=a_{y} \partial \jmath,
$$

por tanto,

$$
\frac{\partial}{\partial x}=\frac{1}{a_{x}} \frac{\partial}{\partial \imath} \quad \text { y } \quad \frac{\partial}{\partial y}=\frac{1}{a_{y}} \frac{\partial}{\partial \jmath}
$$

aplicando a la función de onda, obtenemos

$$
\begin{aligned}
\left.\frac{\partial \psi}{\partial x}=\frac{1}{a_{x}} \frac{\partial \psi}{\partial \imath}=\frac{1}{a_{x}} \frac{\psi_{i+1, j}-\psi_{i-1, j}}{[i+1}-(i-1)\right] & \\
= & \frac{1}{2 a_{x}}\left[\psi_{i+1, j}-\psi_{i-1, j}\right]
\end{aligned}
$$




$$
\begin{aligned}
&\left.\frac{\partial \psi}{\partial y}=\frac{1}{a_{y}} \frac{\partial \psi}{\partial \jmath}=\frac{1}{a_{y}} \frac{\psi_{i, j+1}-\psi_{i, j-1}}{[j}+1-(j-1)\right] \\
&=\frac{1}{2 a_{y}}\left[\psi_{i, j+1}-\psi_{i, j-1}\right] .
\end{aligned}
$$

La corriente de probabilidad se describe de forma discreta como

$$
\begin{gathered}
J_{x}=\frac{\hbar}{2 m^{*} a_{x}}\left(\operatorname{Im}\left[\psi_{i, j}^{*}\left(\psi_{i+1, j}-\psi_{i-1, j}\right)\right]\right), \\
J_{y}=\frac{\hbar}{2 m^{*} a_{y}}\left(\operatorname{Im}\left[\psi_{i, j}^{*}\left(\psi_{i, j+1}-\psi_{i, j-1}\right)\right]+\right. \\
\left.4 \pi \imath \frac{\Phi}{\Phi_{e}}\left|\psi_{i j}\right|^{2}\right),
\end{gathered}
$$

donde $\imath$ representa el índice de sitio en la dirección $x$.

\section{Resultados y discusión}

Para establecer una metodología de análisis de los espectros de energía de los dispositivos que hemos simulado, presentamos en primera instancia el espectro de energía de una red rectangular de $80 \times 55$ sitios que emula el fondo de la banda de conducción del GaAs, Fig 3 , para ello hemos usado como energía de los orbitales tipo $s$ el valor de $E_{i j}=4 V_{0}$ para todos los sitios de la red hospedera y un parámetro de hopping para los primeros vecinos de $V_{x}=V_{y}=V_{0}=0.1422 \mathrm{eV}$, mientras que, el rango de energía está entre 0 y $0.05 \mathrm{eV}$ en la ordenada y el rango de campo magnético relativo $p / q=\Phi / \Phi_{e}$ va desde 0 hasta 0.02 en la abscisa.

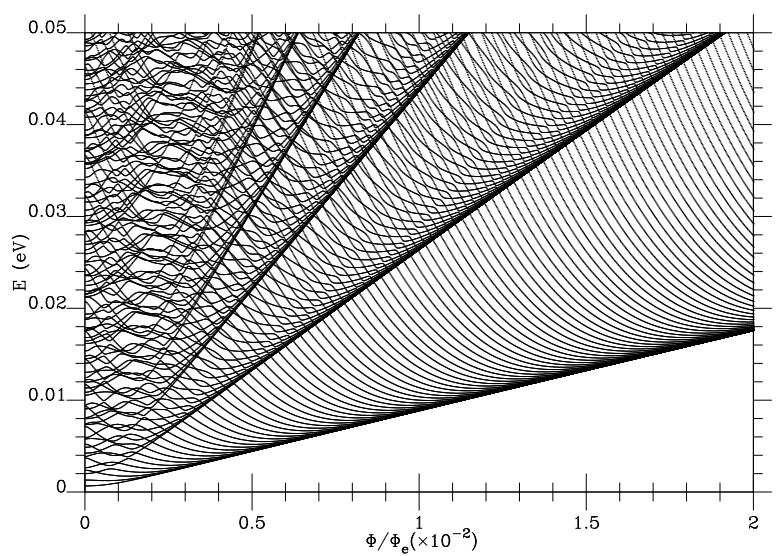

Figura 3: Espectro de energía de una red rectangular de $80 \times 55$ sitios que emula el fondo de la banda de conducción del GaAs.

Se observa la formación de los niveles de Landau con diferentes pendientes conforme el número de Landau aumenta y cómo los estados de borde evolucionan al aumentar el campo magnético localizándose en el nivel de
Landau de número cuántico inferior, hasta llegar al número cuántico $n=1$ debajo del cual no se observa más estados de la caja cuántica rectangular formada por los $80 \times 55$ sitios heurísticos. Este espectro muestra la parte inferior izquierda de la mariposa de Hosftadter [13.

En seguida procedemos a emular, una red hexagonal de antipuntos tal como se muestra en la Fig. 2 donde la forma de cada antipunto se muestran en la figura 4. Para los antipuntos, los sitios en negro poseen la energía $E=16 V_{0}=2.272 \mathrm{eV}$, mientras que, la energía de los sitios de la red hospedera es $E_{0}=4 V_{0}=0.568 \mathrm{eV}$. La figura 4 muestra dos antipuntos, el de la izquierda formado por una red cuadrada de $11 \times 11$ sitios y el de la derecha por una red cuadrada de $5 \times 5$ sitios. El presente reporte está basado en los resultados obtenidos en el antipunto de la derecha.
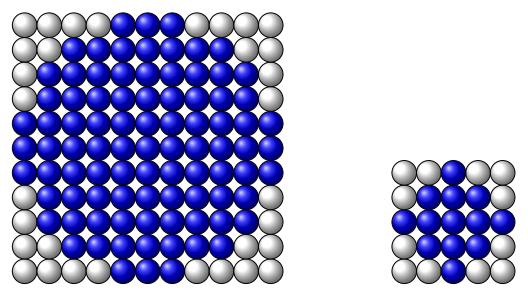

Figura 4: Forma de los antipuntos de $11 \times 11$ (izquierda) y $5 \times 5$ (derecha) sitios heurísticos (azules) respecto al fondo que emula la banda de conducción del GaAs (blancos).

El espectro que corresponde a la primera minibanda de una red de $4 \times 5$ hexágonos de antipuntos cuánticos con cada antipunto conformado por una red cuadrada de $5 \times 5$ sitios heurísticos se muestra en la figura 5 Esta primera minibanda de bulk corresponde a los 32 puntos cuánticos que se distribuyen en pares dentro de cada hexágono de antipuntos tal como se muestran en la Fig. 2

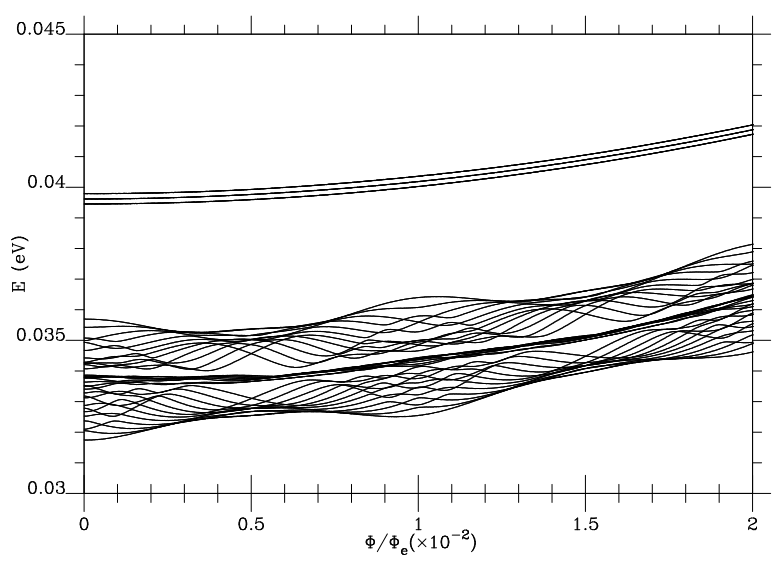

Figura 5: Espectro de energía de la primera minibanda de bulk de una red de $4 \times 5$ hexágonos de antipuntos formado sobre una red hospedera de $80 \times 55$ sitios heurísticos que emula el fondo de la banda de conducción del GaAs. 
Los 32 puntos cuánticos de bulk están distribuídos formando pares de puntos cuánticos (blancos) rodeados de una red hexagonal de antipuntos (azules), Fig 2 En el rango de campo magnético relativo $\Phi / \Phi_{e}$ en que se muestra la figura 5 observamos que la primera minibanda es un espectro autosimilar y emula a la mariposa de Hosfstadter [13] con dos periodos, la primera entre 0 y 0.01 , la segunda entre 0.01 y 0.02 . Esta periodicidad de la mariposa de Hofstadter está asociada con el nuevo periodo espacial de la red de antipuntos hexagonales que se aproxima a $100 \AA$. Puesto que la red hospedera heurísticamente tiene un parámetro de red de $20 \AA$, valor considerado para determinar el parámetro de hopping usado en los cálculos usando la Ec.(11), $V_{0}=0.1422 \mathrm{eV}$.

El primero y el trigésimo segundo de los autoestados de la primera minibanda de bulk se aprecian en la figura 6 , para un campo magnético asociado de $p / q=0.01$, se muestran la densidad de probabilidad en color rojo que representa los valores más altos y en color azul los más bajos y el flujo de la corriente de probabilidad en vectores.
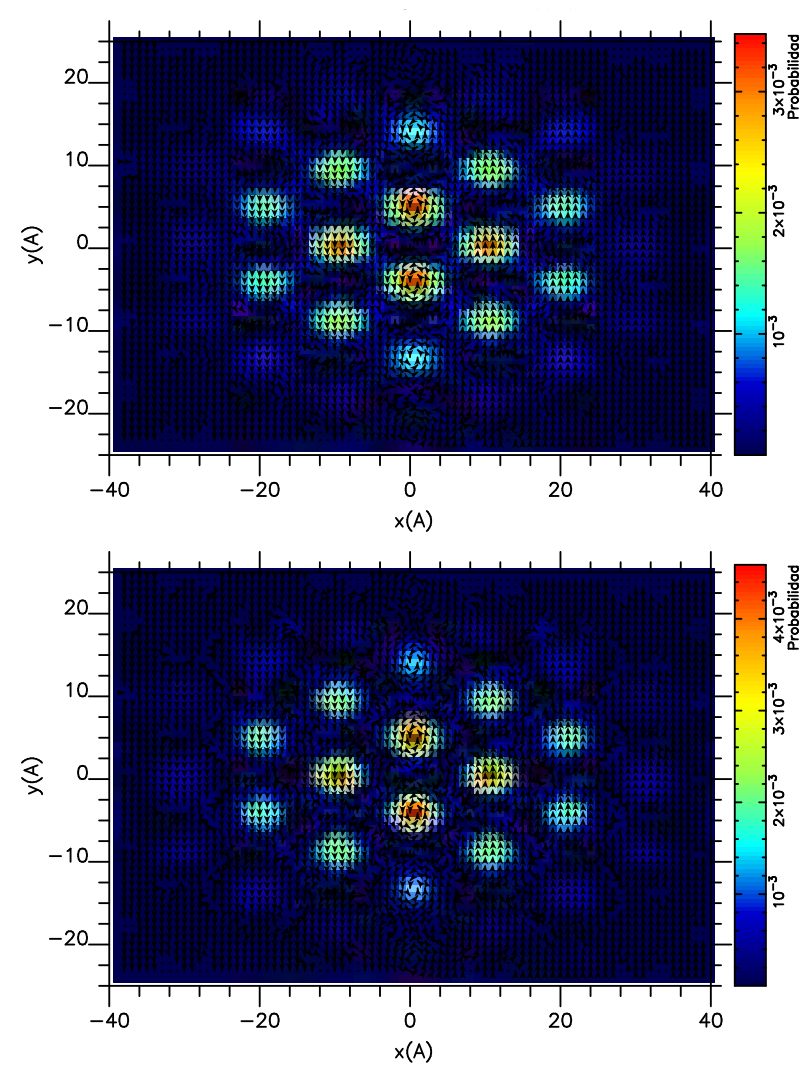

Figura 6: El primer y el trigésimo segundo autoestados correspondientes a la primera minibanda de bulk que corresponde a 32 los puntos cuánticos distribuídos por pares (valles) entre las $4 \times 5$ redes hexagonales de antipuntos. $E_{1}=32.55 \mathrm{meV}$, $E_{32}=36.40 \mathrm{meV}$ y $p / q=0.01$.
Estos dos autoestados $n=1$ y $n=32$ muestran la misma simetría y semejan a un orbital molecular tipo $s$. Continuando el mismo análisis de los autoestados $n=2$ y $n=31$ muestran una simetría de un orbital molecular tipo $p$ y continuando el mismo análisis encontramos que los autotestados $n=12$ y $n=21$ son casi semejantes, pero en la Fig. 5 se observa que el autoestado $n=12$ se acopla fuertemente con el autoestado $n=13$ produciéndose un anticruzamiento.

Analizando los autoestados $n=13$ y $n=20$ se observan que poseen la misma clase de simetría pero la probabilidad de distribuye por todos los puntos cuánticos de manera más homogénea que en los casos anteriores, ver la figura 7.
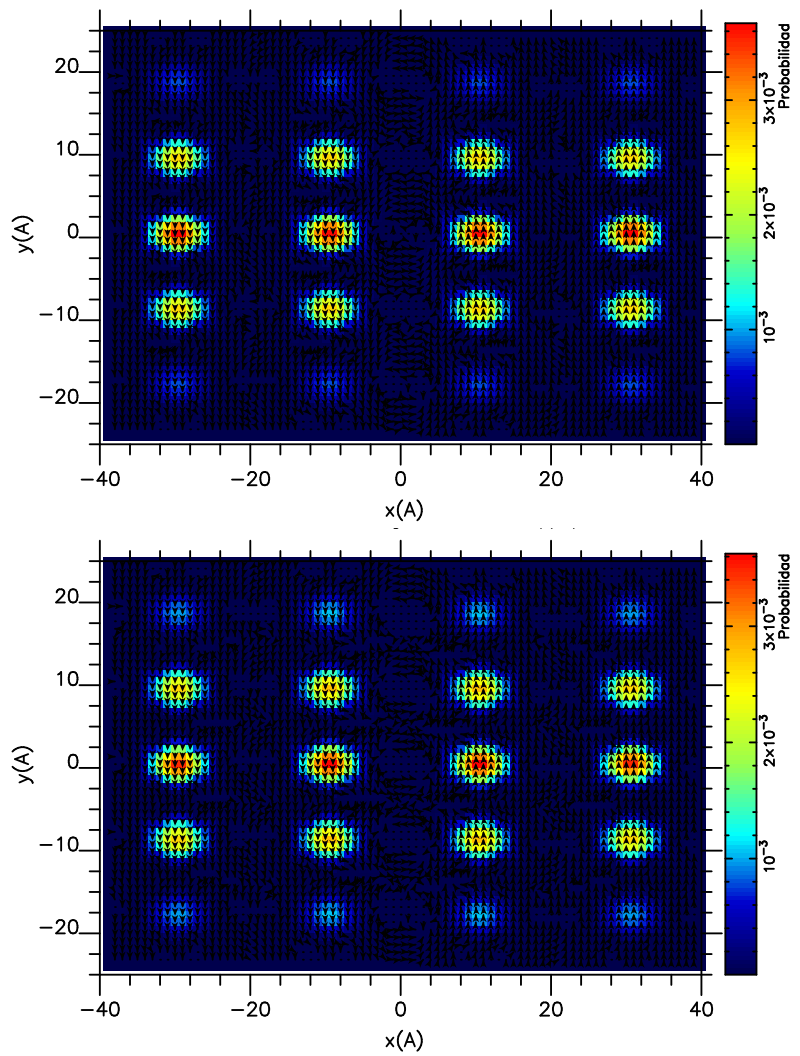

Figura 7: Décimotercero y vigésimo autoestados correspondientes a la primera minibanda de bulk que corresponde a 32 puntos cuánticos formados entre la red de $4 \times 5$ hexágonos de antipuntos. $E_{13}=34.17 \mathrm{meV}, E_{20}=34.44 \mathrm{meV}$ y $p / q=0.01$.

En la figura 7 , se muestran la densidad de probabilidad y la corriente de probabilidad que corresponde a los autoestados $n=13$ y $n=20$ que pertenecen a los estados centrales, ocho autoestados, que evolucionan respecto al campo magnético con cierta independencia a partir de $p / q=0.001$ semejando a una minibanda central e independiente. A esta minibanda de ocho autoestados lo iden- 
tificamos como el punto de Dirac de nuestra red finita de $4 \times 5$ hexágonos de antidots que evoluciona respecto a un campo magnético aplicado, ver figura 5

En la parte superior de la primera minibanda de bulk, a aproximadamente $0.04 \mathrm{eV}$, aparece la evolución de tres estados que son casi doblemente degenerados en la figura 5 pero una revisión de los datos calculados con doble precisión, se observa una quiebra de la degenerescencia de dichos estados a lo largo de todo el intervalo de campo magnético, estos corresponden al acoplamiento de los tres pares de puntos cuánticos que aparecen en la dirección armchair, eje $x$, en la parte superior e inferior de la figura 2 totalizando seis como se puede apreciar en la figura 8 en la que se muestra de densidad de probabilidad y la corriente de probabilidad para el trigésimo tercero y trigésimo octavo autoestado que corresponde a estos estados del borde superior e inferior del dispositivo en la dirección armchair.

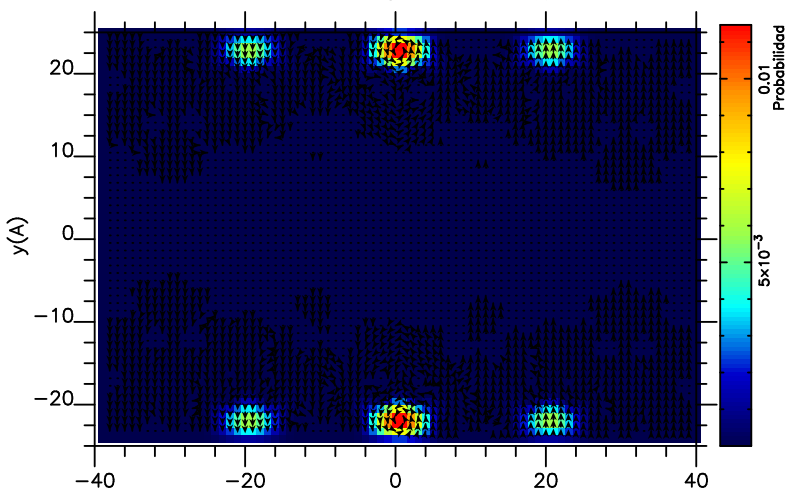

$x(A)$

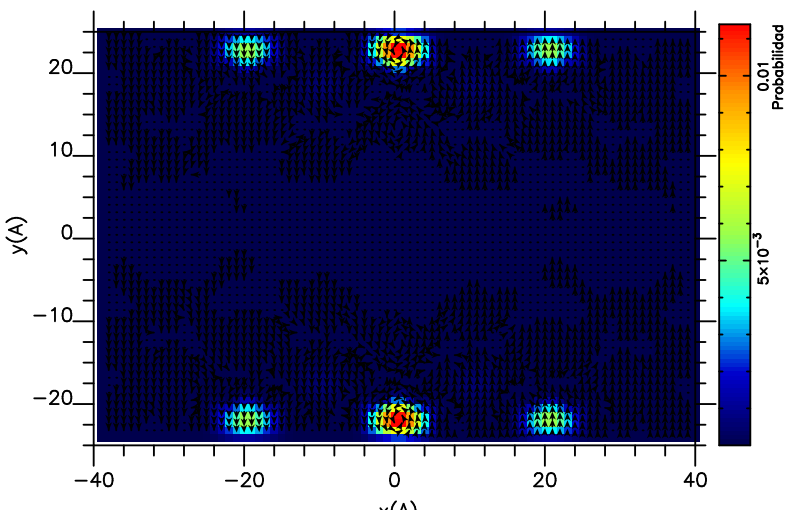

$x(A)$

Figura 8: Trigésimo tercero (superior) y trigésimo octavo (inferior) autoestados correspondiente a los seis autoestados de superficie armchair de la red de $4 \times 5$ hexágonos de antipuntos. $E_{33}=40.03 \mathrm{meV}, E_{38}=40.36 \mathrm{meV}$ y $p / q=0.01$.

Los próximos autoestados que aparecen en el espectro para energía más altas corresponden a la minibanda conformada por los $4 \times 2$ autoestados de superficie en la dirección zigzag, eje $y$, estos evolucionan formando dos pares de autoestados casi-degenerados, que se consigue resolver a nivel de los $\mu \mathrm{eV}$, figura 9 pero se observa que los dos últimos autoestados, $n=45$ y $n=46$, se acoplan con la segunda minibanda de bulk, de modo que los autoestados $n=47$ y $n=48$ son los dos últimos autoestados de superficie zigzag. Después viene la segunda minibanda de bulk que se superponen a los primeros autoestados de canto, a la segunda minibanda de superficie armchair y zigzag, respectivamente. Debido a la gran cantidad de estados que conforman la red hospedera, el ancho de las minibandas, para energías superiores, se incrementa y cubren la presencia de los demás estados de borde y de canto de estos dispositivos que pueden ser determinados continuando este proceso de análisis autoestado por autoestado sistemáticamente.
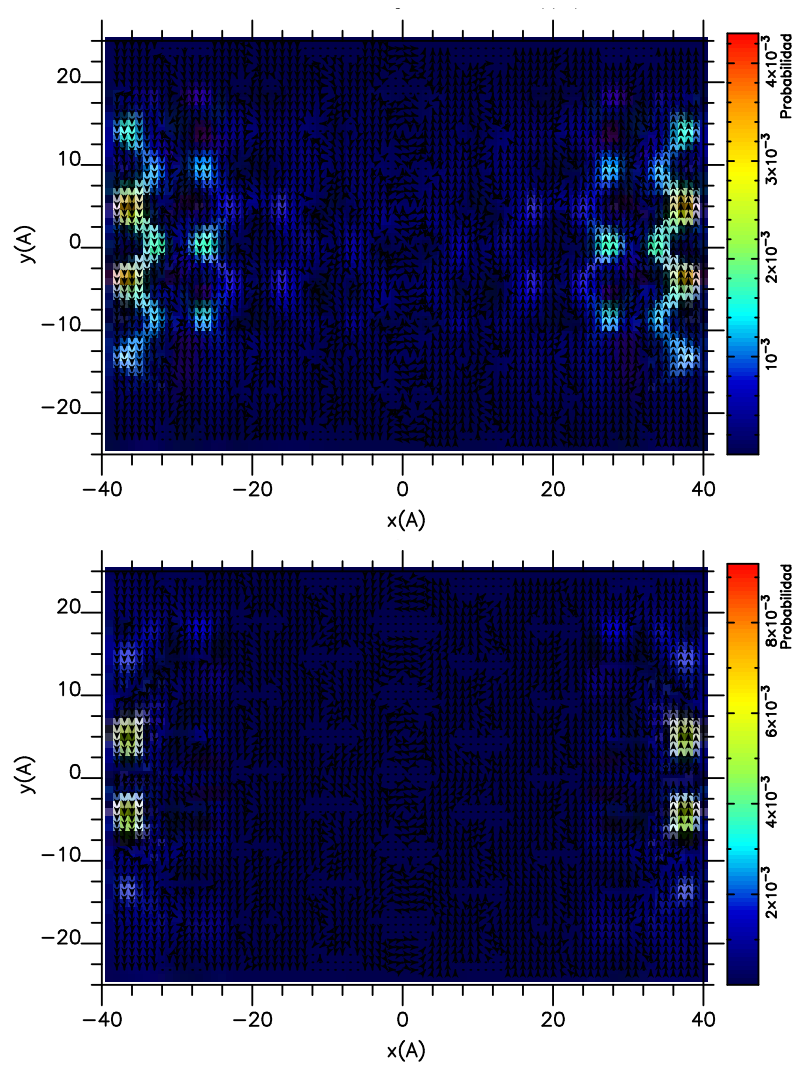

Figura 9: Trigésimo noveno (superior) y cuatrigésimo octavo (inferior) autoestados correspondiente a los ocho autoestados de superficie zigzag de la red de $4 \times 5$ hexágonos de antipuntos. $E_{39}=53.34 \mathrm{meV}, E_{48}=56.55 \mathrm{meV}$ y $p / q=0.01$.

Lo que queda claro de esta discusión, es que las minibandas obtenidas por la construcción de una red hexagonal de antipuntos en la banda de conducción del GaAs, corresponden a la estructura de una red hexagonal de puntos cuánticos, cuya primera minibanda es identificable me- 
diante las figuras 6 y 7, que muestran las densidades de probabilidad y las corrientes de probablidades correspondientes.

Los estados de borde que en última instancia son los responsables del transporte electrónico para nanodispositivos son identificables y están aislados de la primera y las demas minibandas. Obviamente el potencial químico de los contactos que se acoplen a estos dispositivos deben estar en torno de 39 y $42 \mathrm{meV}$ en el rango de campos magnéticos escogidos para el presente trabajo, $p / q: 0 \rightarrow 0.01$.

El punto $\mathrm{K}$ y $\mathrm{K}^{\prime}$ para esta estructura hexagonal corresponde a los 8 autoestados de bulk que evolucionan de manera independiente respecto a los otros 24 autoestados de la primera minibanda de bulk, esto también se aprecia para la segunda minibanda de bulk.

Por tanto, las propiedades de transporte electrónico que corresponde a este diseño de dispositivo se basa solo en las propiedades de transporte del GaAs y la performance son propias de las propiedades del GaAs próximos al punto $\Gamma$.

\section{Referencias}

[1] K. S. Novoselov, A. K. Geim, S. V. Morozov, D. Jiang, M. I. Katnelson, I. V. Grigorieva, S. V. Dubonos y A. A. Firsov; Nature 438, 197 (2005).

[2] Yuanbo Zhang, Yan-Wen Tan, Horst L. Stormer y PhiIlip Kim; Nature 438, 201 (2005).

[3] K. S. Novoselov, A. K. Geim, S. V. Morozov, D. Jiang, Y. Zhang, S. V. Dubonos, I. V. Grigorieva y A. A. Firzov; Science 306, 666 (2004).

[4] M. Fujita, K. Wakabayashi, K. Nakada y K. Kusakabe; J. Phys. Soc. Japan 65, 1920 (1996).

[5] K. Nakada, M. Fujita, G. Dresselhaus y M. S. Dresselhaus; Phys. Rev. B 54, 17954 (1996).

[6] P. H. Rivera, A. L. C. Pereira y P. A. Schulz, Phys. Rev. 79, 205406 (2009).

[7] T. G. Pedersen, C. Flindt, J. Pedersen, N. A. Mortensen, A-P. Jauho y K. Pedersen, Phys. Rev. Lett. 100, 136804 (2008).

\section{Conclusiones}

En una red hexagonal de antipuntos cuánticos litografiado sobre la heteroestructura de GaAs/AIGaAs se obtiene una red hexagonal de puntos cuánticos cuya primera minibanda está aislada de los autoestados que corresponden a los estados de borde. Estos estados de borde son formados en la dirección armchair y la dirección zigzag de la red hexagonal de antipuntos.

Las propiedades de transporte de estos dispositivos se basan en las propiedades del GaAs próximo al punto $\Gamma$. Puesto que una identificación de los autoestados próximos a los puntos $\mathrm{K}_{\text {y }} \mathrm{K}^{\prime}$ de la red artificial hexagonal ha sido posible por la autonomía en su evolución de los ochos autoestados centrales en la primera y segunda minibandas de bulk en puntos cuánticos hexagonales en función del campo magnético.

\section{Agradecimientos}

Agradecemos el apoyo financiero concedido por el Consejo Superior de Investigaciones mediante el proyecto № 121301041.

[8] C-H Park, L. Yang, Y-W Son, M. L. Cohen y S. G. Louie; Phys. Rev. Lett. 101, 126804 (2008).

[9] A. Singha, M. Gibertini, B. Karmakar, S. Yuan, M. Polini, G. Vignale, M. I. Katsnelson, A. Pinczuk, L. N. Pfeiffer, K. W. West, V. Pellegrini, Science 332, 1176 (2011).

[10] L. Nádvorník, M. Orlita, N. A. Goncharuk, L. Smrčka, V. Novák, V. Jurka, K. Hruška, Z. Výborný, Z. R. Wasilewski, M. Potemski y K. Výborný, arXiv:1104.5427v.2 (2011).

[11] K. Ensslin y P. M. Petroff, Phys. Rev. B 41, 12307 (1990).

[12] R. Schuster, K. Ensslin, D. Wharam, S. Kühn, J. P. Kotthaus, G. Böhm, W. Klein, G. Tränkle y G. Weimann; Phys. Rev. B 49, 8510 (1995).

[13] D. Hofstadter; Phys. Rev. B 14, 2239 (1976). 